\title{
Deploying Advanced IP Services on a Community Network
}

\author{
João Paulo Firmeza, Francisco Fontes \\ Portugal Telecom Inovação, SA \\ Rua Eng. José Ferreira Pinto Basto, 3810 Aveiro, Portugal \\ \{pfirmeza, fontes\}@ptinovacao.pt \\ Tel: +351 234403311, Fax: +351 234420722
}

\begin{abstract}
Community Networks have been implemented for the last few years on several cities, most of them supported by local governments or by experimental projects. Portugal was not an exception and since the early 1990 s some small-scale projects were deployed. By the end of 1998, the Portuguese government sponsored a new wave of community networks under the scope of the project 'Digital Cities', promoting the use of new technologies and the development of enhanced services for the citizen. The city of Aveiro was the first of a new generation of Digital Cities in Portugal. This paper describes, in detail, network implementation options for this community network, concerning both its core and access enabling technologies, as well as the services that are being provided, including description and architectures for each of the relevant ones.
\end{abstract}

\section{Introduction}

Aveiro Digital City is the name of a large-scale community network sponsored by Portugal Telecom and by the Portuguese government, located in the region of Aveiro, $60 \mathrm{~km}$ in the south of Oporto. To support this community, an IP multi-services platform was set-up, requiring services ranging from typical IP narrowband to broadband and real-time services to coexist peacefully with the best possible quality.

All those services are supported by a common platform providing users with a set of basic services such as a portal service, e-mail, Web hosting (both institutional and personal pages), personalization services, discussion forums, IRC, multi-point H.323 conferences, and games. For users and projects with special needs a set of advanced services is also available like an open e-commerce platform, broadband IP streaming (MPEG1/2 VoD streaming and IP based Interactive TV) and high bitrate H.323 conferencing services.

This community network in place for almost one year and will grow to support in the near future more than 20.000 users, mostly accessing via Plain Old Telephony System (POTS), Integrated Services Digital Network (ISDN) and Asymmetric Digital Subscriber Line (ADSL). Very high speed Digital Subscriber Line (VDSL) and FibreTo-The-Home (FTTH) technologies are also available from a local trial and are being 
used to connect a number of special users. Core network technology is based on Asynchronous Transfer Mode (ATM) switches and IP routers.

Based on the experience obtained with Aveiro Digital City, and supported by its success, new similar experiences will grow in Portugal during the next few years. Besides improving existing Digital Cities, new ones will require the integration of new services and network technologies, based on particular user profiles and environment characteristics.

\section{The Network and Its Basic Services}

\subsection{The Core Network}

Core network was defined in order that bandwidth limitations should not become a problem, at least in the medium term. For that purpose, Ethernet and ATM network technologies were considered and evaluated in terms of advantages and disadvantages. Ethernet $(100 \mathrm{Mbit} / \mathrm{s}$ and $1 \mathrm{Gbit} / \mathrm{s})$ is a cheap and simple technology that offers very good performance, but it is a pure local area network (LAN) technology, not suitable for a wide area network (WAN) application and for interconnection scenarios (very likely to exist in different flavours on our network). Additionally, quality-of-service (QoS) management is very limited.

On the other hand, a pure ATM solution is more complex, more expensive and less performing when used in a LAN environment, but it offers very good QoS mechanisms and is very well suited for the required interconnection scenarios. Moreover, ATM is required as transport technology for some of the available access technologies that were intend to be used, such as ADSL and VDSL.

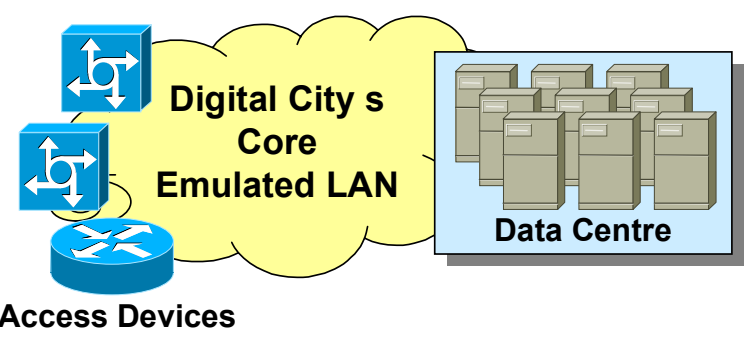

Fig. 1. The Core ELAN and connected devices: Data Centre Services and Access Devices

The final adopted solution was a hybrid one: switched and dedicated Fast Ethernet (100 Mbit/s) on the server farms and ATM for the core and access device interconnection. Since all the services are IP based, IP is the only protocol supported on this network, over Ethernet and ATM technologies.

On this hybrid core network, an ATM emulated LAN (LANE) was established, thus allowing the seamless integration of Fast Ethernet hosts and native ATM 
equipment over a single infrastructure. For this, a Cisco Catalyst 5500 switch equipped with one redundant ATM STM-4 card and a router switch module was used in the core network. This LANE is the main 'cloud' of Aveiro's Digital City network; the services data centre and some of the access/edge devices are directly connected to this cloud over ATM or Fast-Ethernet. ATM connected access devices reach the Emulated LAN through a Cisco LS1010 ATM switch (Figure 1). Besides the core LANE-based virtual LAN (VLAN), other VLANs are configured on this network: one for service management, one to support a special project and several to support RFC1483 bridged ADSL clients, as described later on. The Catalyst route switch module assures IP routing between those VLANs. A schematic diagram of the core network is shown in Figure 2.

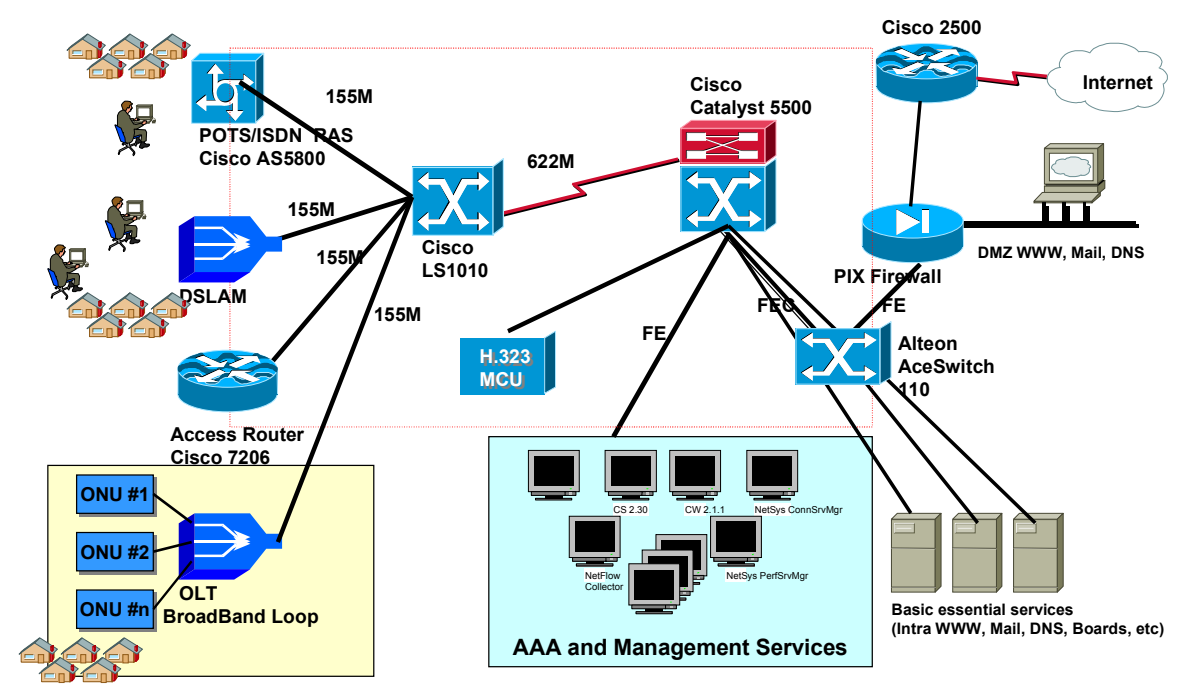

Fig. 2. Aveiro Digital City's Network Diagram

\subsection{Access Infrastructure}

This network supports several access options, narrowband and broadband. Narrowband access comprises POTS and ISDN dial-up at rates up to $56 \mathrm{kbit} / \mathrm{s}$ on analog modems or up to $128 \mathrm{kbit} / \mathrm{s}$ on ISDN. A Cisco 5800 access server, featured with 12 primary rate lines and 10 MICA modem cards, is being used to terminate up to 720 simultaneous PPP sessions. User authentication and accounting uses a Lightweight Directory Access Protocol (LDAP) enabled Remote Authentication Dial In User Service (RADIUS) server, which is part of the service platform described later on. Broadband access is provided through three different technologies: ADSL, VDSL and FTTH. 
ADSL service is being provided by two Digital Subscriber Line Access Multiplexer (DSLAM), one from Alcatel and one from Siemens. They are located in the Aveiro's central telephone office.

The service is provided in 2 different configurations, according to particular requirements. Some users/projects use ADSL in an always-on basis; in this case IP services use RFC1483 bridged over ATM PVC connections terminating on the core switch, which comprises a VLAN for each of these user groups/projects (Figure 3a). This is the case of a kiosks project described later on.

Other users/projects are using PPP/PPPoA/PPPoE ${ }^{\natural}$ sessions terminated on a BroadBand Remote Access Server (BBRAS). In this case PVCs carrying PPP sessions are established from user premises ATU-R devices and terminated on the BBRAS. Those sessions are authenticated using the same AAA server as for the narrowband users (Figure 3b).

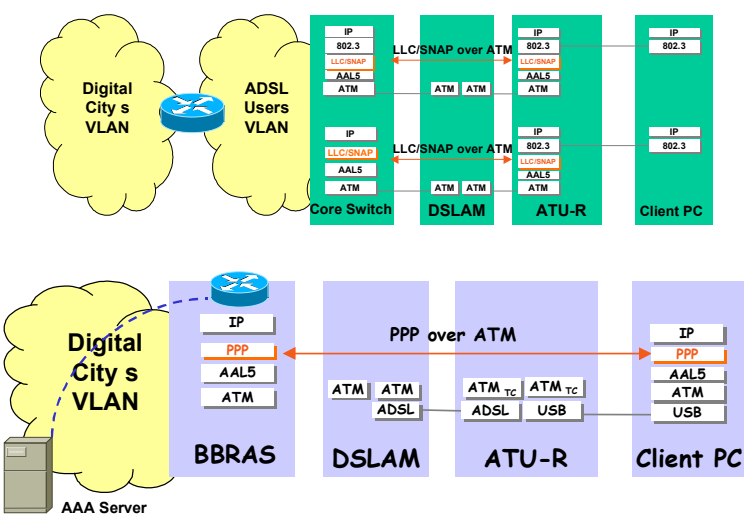

Fig. 3. Diagram of ADSL access scenarios. a) Access using RFC1483 bridged b) Access using $\mathrm{PPP} / \mathrm{PPPOA}$

For users connecting through ADSL, special broadband services are available on the network, such as Video-on-Demand or local IP-based television programmes, as shown in Figure 4.

VDSL and FTTH technologies are supported by the BroadBand Loop project's field trial in Aveiro. BroadBand Loop (BBL) was a project supported by the EU ACTS programme (AC038). It addresses access network solutions based on ATMpassive optical network (ATM-PON)/VDSL technology, paving the way for the widespread usage of broadband services in the local loop. As an evolution step from narrowband topologies towards broadband service features, BBL followed a hybrid fibre-copper approach.

\footnotetext{
${ }^{1}$ PPPoA: PPP over ATM. Defined in RFC 2364

PPPoE: PPP over Ethernet: Defined in RFC 2516
} 

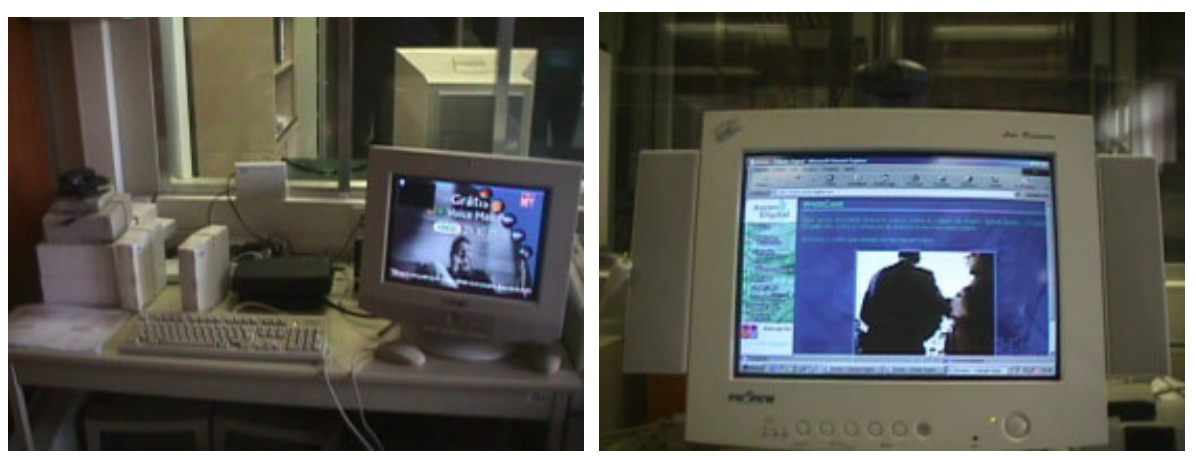

Fig. 4. Video-On-Demand and High-bitrate video streaming integrated on Digital City's service Portal using an ADSL access.

The Portuguese field trial system installed in Aveiro is based on a hybrid fibre/copper transport system providing end-to-end ATM transport. The optical distribution network (ODN) is based on a PON network comprising a single head-end unit, the optical line termination (A-OLT) with 16 remote optical network units (ONUs) located in street cabinets or directly on the customer's premises. A reference architecture is shown in Figure 5 and Interface definitions are given in the Table 1.

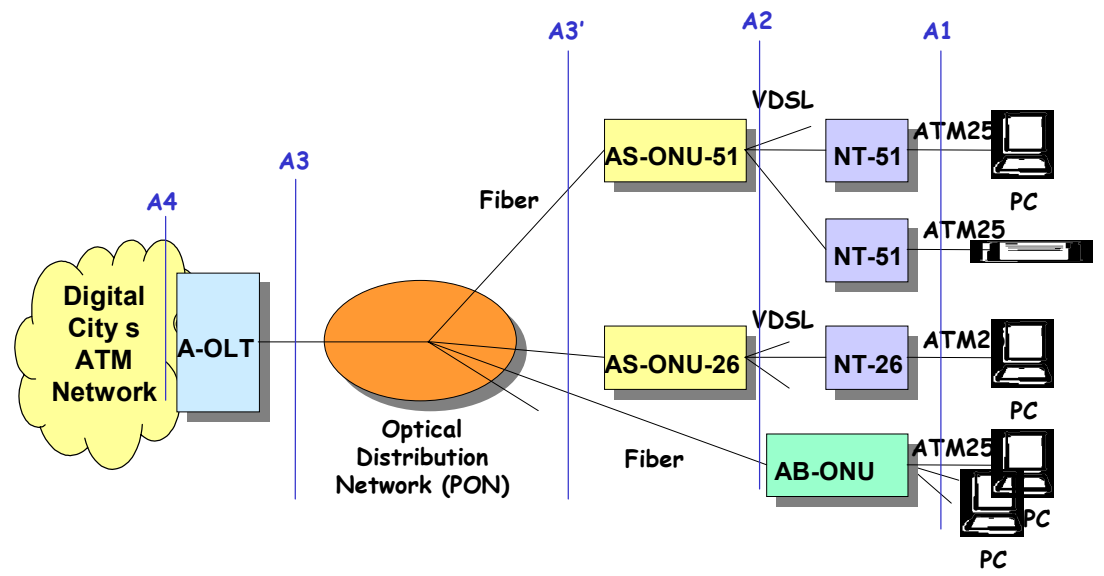

Fig. 5. A PON reference architecture

Whenever the ONU is located in a street cabinet, the last drop to the customer is based on two forms of VDSL technology supporting short-range $(200 \mathrm{~m})$ or medium range $(800 \mathrm{~m})$ data transfer at fixed bandwidths. The VDSL lines are terminated using an active network termination (NT) function supporting up to two ATM-Forum compliant ATM-25 interfaces. ONUs and NTs are named on the basis of their VDSL delivery capabilities. An AS-ONU-26 supports four VDSL 26/3 (Mbit/s) lines, each terminated by an NT-26, whereas an AS-ONU-51 supports eight VDSL 51/1.6 (Mbit/s) lines each terminated by an NT-51. In all other situations, where the ONU is located in the customer's premises, a business ONU (AB-ONU) supporting a direct 
fibre termination and two ATM-25 interfaces is deployed. AS-ONUs are used in fibre-to-the-cabinet (FTTC), fibre-to-the-kerb (FTTK), and fibre-to-the-exchange (FTTE) topologies and AB-ONUs are used in a fibre-to-the-home (FTTH) topology.

Table 1: PON architecture Interface definitions

\begin{tabular}{|l|c|}
\hline A1 & ATM-25 \\
\hline A2 & VDSL-51, VDSL-26 \\
\hline A31 & Split fibres \\
\hline PON downstream & VC-4 (STM-1, G.707) \\
\hline PON upstream & TUG-A (proprietary) \\
\hline A3 & Single fibre \\
\hline A4 & STM-1 or STM-4 \\
\hline
\end{tabular}

Figure 6 shows a BBL AS-ONU mounted on a street cabinet and a VDSL modem (NT-51). The entire BBL network consists of 16 ONUs, 10 of them are AB-ONUs, two are AS-ONU-51 and four are AS-ONU-26s, giving a total capacity of connecting 42 clients (NT-26s and AB-ONUs provide to each one 2 ATM-25 ports).
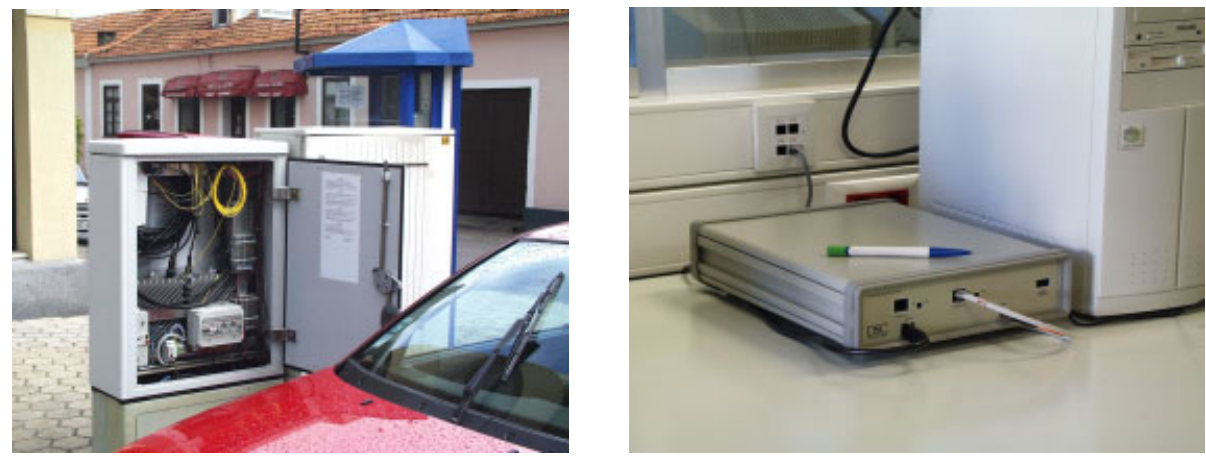

Fig. 6. BBL AS-ONU mounted on a street cabinet and a VDSL modem (NT-51).

IP services are being delivered over ATM PVCs via optical line terminations (OLTs) on the ATM switch to Aveiro Digital City's ATM core network. Since bandwidth limitation was not a problem on the PON network, all the connections were configured with constant bit rate (CBR) class of service, allowing the most sensitive services, such as MPEG2 $\mathrm{VoD}$ and $\mathrm{H} .323$ videoconferencing to behave properly. Each of the connections has a peak cell rate of $20 \mathrm{Mbit} / \mathrm{s}$ on each of the client ATM-25 adapters and traffic shaping was applied to limit upstream bandwidth ( $3 \mathrm{Mbit} / \mathrm{s}$ for the NT-26, $200 \mathrm{kbit} / \mathrm{s}$ for the NT-51 and $9 \mathrm{Mbit} / \mathrm{s}$ for the AB-ONU).

IP transport uses IP-over-ATM (RFC1483) routing. For each ONU drop point, an IP subnet was assigned; each one of these subnets has a virtual interface defined on the access router allowing each user's traffic to be routed to the core network, from where services are being provided. 


\subsection{Data Centre Service Architecture and Generic Services}

A generic IP services platform provides the building blocks for all the services developed for this community. This platform was designed with the objective to require a minimum effort to develop any specific high-level service for any specific sub-community or project. This main goal led to the following sub-objectives:

- Simplified process of user registration and automatic provisioning of customised services.

- Reduced end-user administration, operations, and support costs.

- Easy creation and deployment of new services.

- Easy and decentralised server management.

The generic service platform and its supporting information system were implemented using both Microsoft Commercial Internet Services (MCIS) and LINUX based open-source solutions. A particularly important service is the personalisation and membership directory and its back-office database. The membership directory provides a LDAP based hierarchical directory optimised for storing the whole of service's and user's information.

The membership directory can be used to store user properties that are leveraged by the personalisation features of MCIS. In addition, Digital City services use the membership directory to store user authentication credentials and permissions as well as other information required for some community services. With the membership the following features are available:

- extensible LDAP schema, allowing creation of new attributes for existing users or to define entirely new classes of directory objects;

- LDAP interface with a well-known service interface (Active Directory Services Interface (ADSI)) that enables easy access to directory objects and attribute manipulation;

- a directory security model.

Authenticated services, such as remote dial-up accounts, e-mail accounts, protected web-content, or user accounts for content uploading, are all supported by the membership directory authentication service. Standard services such as WWW, email, RADIUS and file transfer protocol (FTP) can use the membership as an authentication provider through membership's LDAP interface. Figure 7 illustrates the membership's architecture.

Client

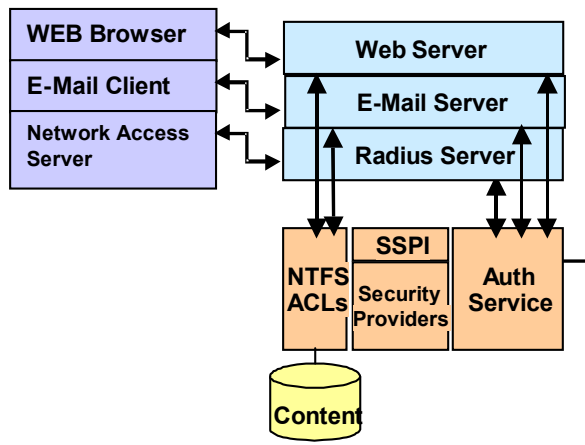

Directory Server

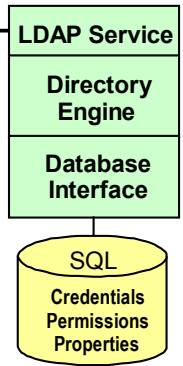

Fig. 7. Membership Architecture 


\subsubsection{Information System Integrated Services}

The basic services provided by the IP platform are: web hosting, Internet relay chat, web-based discussion boards, software archive, H.323 conferencing, video streaming and AAA. Additionally RADIUS, e-mail (SMTP, POP3 and IMAP4), personal web pages, and some protected web based services (like web based front end for e-mail service) are available, using authentication based on the membership authentication provider using its LDAP interface.

Taking as an example the case of the e-mail service, the interaction with the membership and storage area where user mailboxes are located is done like this: when a client tries to access its POP3 or IMAP4 server, the server authenticates the user against the membership directory to verify if the user can be logged on. If the authentication is successful, another LDAP query returns the location of the user's mailbox directory. The mailbox can either be located in a local server directory or on a remote file store. A SMTP server will accept mail for local delivery (inbound) from any host from which it accepts an SMTP connection. For outbound mail, the SMTP server can be set up to accept only connections from authenticated users, from specific IP-address ranges or both, in order to cut down spamming.

Figure 8 shows the basic architecture of the Digital City's Data Centre.

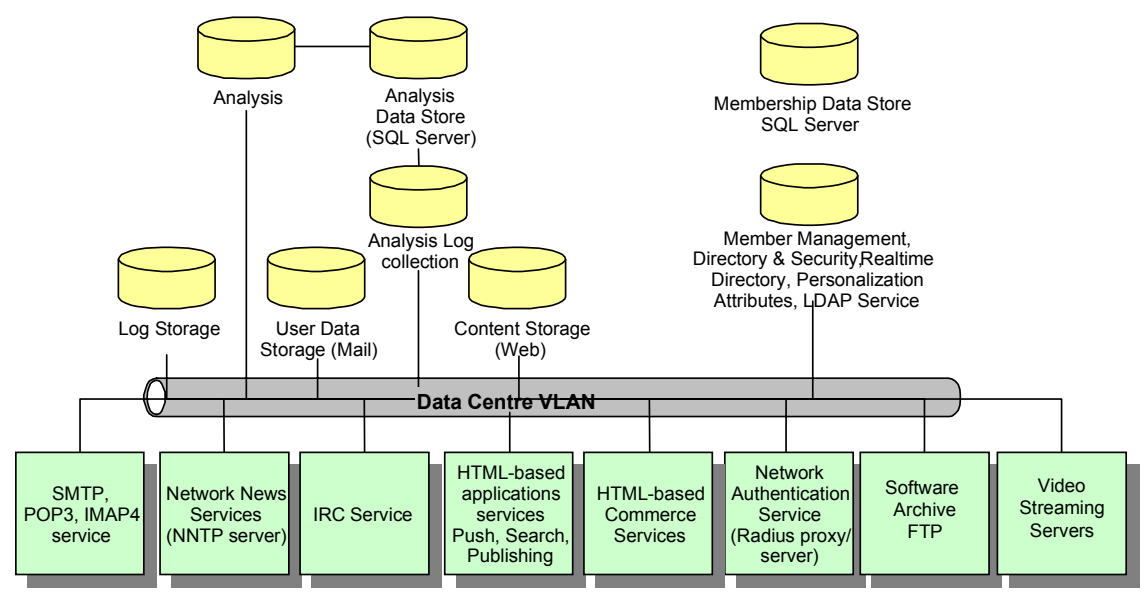

Fig. 8. Data Centre Service Architecture

\subsubsection{Internet Access Service}

From the point-of-view of the community network, Internet access is just another service. Internet is accessible via a firewall placed between Digital City's VLAN and a small unprotected LAN. Both network address and port address translation (NAT and PAT) are used to translate private IP addresses from the community network to public IP addresses assigned to Aveiro Digital City. In addition, a demilitarised zone is used to place some Internet services, like the public Domain Name System (DNS) or public Web servers. To increase performance and conserve bandwidth on the Internet connection (a E1 connection), Internet Web access is made through a transparent proxy/caching service. 
A traffic redirector was configured on the router that handles traffic from Digital City's intranet to the Internet firewall; all hypertext transfer protocol (HTTP) requests from inside to external addresses are sent to the transparent proxy/cache servers. Caching service is implemented using Squid cache software running on two Linux boxes, which are placed beyond a L4 switch from Alteon Web Systems. This switch can load balance Web requests to the existing cache servers.

\subsubsection{Multi-point Conferencing Service}

To support multi-point audio/video conferences, an H.323 MCU is available on the data centre. This device is configured to be running, permanently, a free access conference. Like other services, the conferencing service uses the same LDAP based directory platform, allowing users to be registered when they are available for conversation. Conference names are also registered on the directory.

To manage closed conferences, a web-based conference management interface is being developed allowing special users with the right privileges to create, edit and view those conferences on the conferencing system.

Typical clients for this service are using Microsoft NetMeeting 3.0 for low quality conferencing and Intel ProShare conferencing kits for high quality conferences.

\section{Projects and Enhanced Services}

Apart from supporting generic IP services, this network is also the place where platforms and specific content belonging to some of the projects running on the Digital City initiative are located. In some cases, special services or extensions to existing platforms, were developed to support these projects. Projects on the Digital City are segmented in terms of intervention area, such as public information services, school and education communities, municipality support services, health care services, services for commerce and industry and social exclusion aid services.

Targeting those intervention areas, Aveiro Digital City initiative has currently 40 projects running. Four of these projects were selected for a briefly description in the next sections, based on the particular services that were developed to support them or on the special features implemented over the common network.

\subsection{Project EIRÓ}

EIRÓ is a project that consists of 12 outdoor kiosks, placed around the city, to give citizens and tourists public city information such as vectorial browseable/clickable city maps (based on a geographic information system), weather information, cultural events, local news and tourism information, with high quality videos (MPEG2) and audio.

Those kiosks are connected together and with the core, over the ADSL network, using RFC1483 bridged connections into a special VLAN supported on the core infrastructure. A special server farm located at the data centre building gives 
exclusive services to this VLAN. MPEG-1 and MPEG-2 video streaming up to 6 Mbit/s is being supported on Microsoft NetShow Theater platform, while lower bandwidth video and audio streaming is being provided by Microsoft Windows Media Services platform. User interface and dynamic data is based on an HTTP server and a Structured Query Language (SQL) server. Kiosk Graphical User Interface (GUI) was developed using ASP technologies, eXtensible Markup Language (XML) and Dynamic HyperText Mark-up Language (DHTML). A special user interface was designed for back-office operators allowing them to update the system SQL based information (with news, events, etc) or to update the multimedia library (audio/video).

In addition to typical kiosk functionality, each of these street-mounted public boxes also acts as a public telephone post. This service uses Portugal Telecom's Intelligent Network in combination with voice-over-IP on the Digital City's network. A user owning a virtual calling card (identified by a card number and a PIN) can dial from a public kiosk to any public network telephone number, by using a GUI-based dialer pad. These calls are routed in IP over the project's VLAN, then routed to Digital City's VLAN and finally handled by a H.323 gateway placed on the core network. EIRÓ's network architecture is shown on Figure 9.

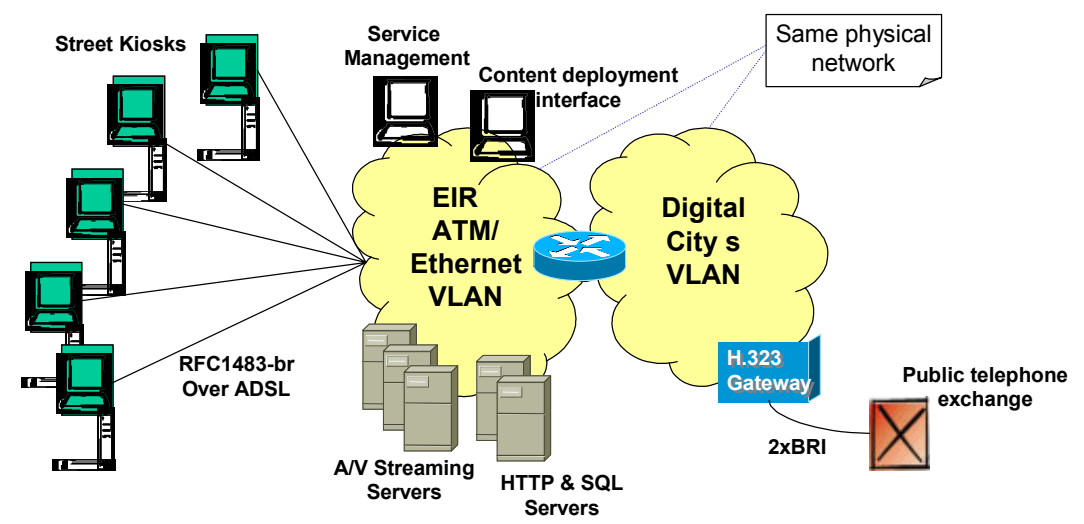

Fig. 9. Project 'EIRÓ' network architecture

\subsection{Project AVEIRO MEGASTORE}

In this e-commerce project a shop for the community network was developed. 18 existing traditional commerce shops participate on this project and are connected to Digital City's core network mostly by ADSL and ISDN dial up. These shops are able to use all the generic services and in addition are able to access the commerce platform to exchange transactions (orderings, payments etc), and also to update their own product catalogues or place promotions. Microsoft Site Server was used to build the e-commerce platform, being used exclusively by the Aveiro Megastore project. However this platform has capacity to support as many e-commerce sites as needed. Secure transactions use Secure Socket Layer (SSL) and are based on certificates issued by an internal Certification Authority (CA). 


\subsection{Project EABL.NET}

This project has built a Virtual Private Network (VPN) for farmers and cow producers in the region of Aveiro. This VPN runs over Digital City's network and uses secure IP-tunnelling technologies (L2TP and IPSec). This network uses a topology where all the farmers connect via ISDN dial-up to a central office where information systems for agriculture and cattle-breeders are located (Figure 10).

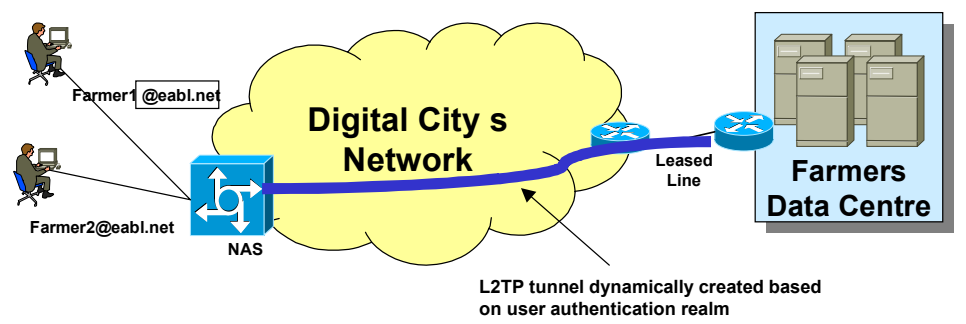

Fig. 10. Project 'EABL.NET' network architecture

\section{Conclusions}

Based on the experience acquired with Aveiro Digital City, and supported by its success, new similar experiences will grow in Portugal during the next few years. A new official programme called Digital Portugal was already approved extending the digital city's concept to the entire country. Besides improving existing Digital Cities, new ones will require the integration of new services and network technologies, based on user profiles and surrounding environment needs. Connection to actual and future broadband ATM and IP back-bones, with a national coverage, will allow the deployment of existing and emerging services based on core network functionality.

Support for user communities services, based on the VPN concept, will allow a coherent distribution of components between individual community network and operator networks. Figure 11 shows the integration of other city communities on Aveiro's common service platform.

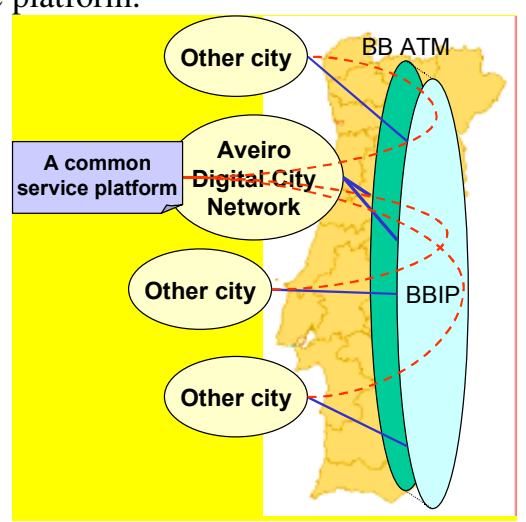

Fig. 11. Integration of other city communities on Aveiro's common service platform 
Reusing Aveiro's platform to host new communities will be our target for the future. In that scenario QoS provision mechanisms and more powerful management procedures must be further developed. This may require the integration of new network solutions, QoS aware, like integrated services and differentiated services, together with monitoring and management tools. The ability to differentiate between different user application areas will allow better usage of network resources. Adopting QoS policies, will distinguish between user services and applications requiring different network levels of commitment.

\section{References}

1. Baldonado, M., Chang, C.-C.K., Gravano, L., Paepcke, A.: The Stanford Digital Library Metadata Architecture. Int. J. Digit. Libr. 1 (1997) 108-121

2. Bruce, K.B., Cardelli, L., Pierce, B.C.: Comparing Object Encodings. In: Abadi, M., Ito, T. (eds.): Theoretical Aspects of Computer Software. Lecture Notes in Computer Science, Vol. 1281. Springer-Verlag, Berlin Heidelberg New York (1997) 415-438

3. van Leeuwen, J. (ed.): Computer Science Today. Recent Trends and Developments. Lecture Notes in Computer Science, Vol. 1000. Springer-Verlag, Berlin Heidelberg New York (1995)

4. Michalewicz, Z.: Genetic Algorithms + Data Structures = Evolution Programs. 3rd edn. Springer-Verlag, Berlin Heidelberg New York (1996) 